\title{
A study of production and consumption of information graphics in Indian regional newspapers
}

\author{
Udayan Vidyanta* \\ IDC School of Design, IIT Bombay
}

\author{
Venkatesh Rajamanickam ${ }^{\dagger}$ \\ IDC School of Design, IIT Bombay
}

\begin{abstract}
This work involved collecting the infographic data for the month of December 2017 from the Delhi editions of top four most-read Hindi newspapers and top four most-read English newspapers in India $[1,15,24]$. This data is organised newspaper-wise, and date-wise with certain parameters of judgement like the content it is depicting, the location of the news in the newspaper, its treatment, and the area it covers on a page. We propose a tentative data model for a systematic collection of the otherwise unusable infographic data, in a database, ready for analysing and deriving insights. This model can be used to gather similar data from other regional languages (Bangla, Tamil, Malayalam, etc.) newspapers printed in India.
\end{abstract}

Index Terms: Human-centered computing-Visualization-Visualization techniques - Infographics; Human-centered computingVisualization-Visualization design and evaluation methods

\section{INTRODUCTION}

The data presented in newspapers all across the world, in the form of various news articles, can be a rich source for analysing what type of news is considered as important for the people, whether a particular newspaper is biased towards a particular category of news, etc. The factors like the form in which the news is presented, the location of the data in the newspaper, the percentage size of the page that is allocated to the news, and the colour treatment of the news article show the importance given by the newspaper to that particular news. This data is lying unused in various newspaper archives since it seems to not be in a format fit for analysis. No work has been done to identify methods fit for collection and organisation of this data.

Infographics are a great storytelling tool to be used in newspapers. They take typically dry data, text-based information and present it in a visual manner. They are concise, easily-digestible, and aesthetically appealing, incorporating clever visual elements to highlight key information. The key goal of infographics is to provide the audience with a piece of short sharp information in a way that is memorable [18]. They combine the best of text, images and design to deliver a story to the readers in an appealing and memorable way, saving space at the same time [25]. Infographics are generally considered a metric for the reader base's composure. It requires a certain amount of skill and patience to read and understand data visualisation. Therefore, the amount of news represented as infographics is considered a direct representation of how sophisticated the reader base is. Establishing methods for collection of this type of data and rendering it in a form fit for analysis, was the key goal of this project.

This project only hits the surface while collecting the infographic data from the newspapers only for the month of December 2017. However, in the process, it establishes a tentative data model for collecting and organising this type of data. This work highlights

*e-mail: udayan.vidyanta@gmail.com

†e-mail: venkatra@iitb.ac.in problems in collecting this type of data, different approaches taken to standardise the process for data collection, and the rationale used for organising this data. We started this project as a pilot to a larger project, with the intention of standardising a procedure for data collection and organisation. The pilot was conducted in the month of December 2017 for two reasons. Firstly, December was supposed to be the month of assembly elections in the Indian state of Gujarat and secondly, the newspapers in the last month of the year populate summary of the important events that happened over the year. We thought that there would be a lot of visualisations in December to collect. A set of English and Hindi dailies was chosen to get a comparison of the quality and quantity of visualisation used in the two languages. We chose Hindi dailies to be compared against English because of our native proficiency in the language. Though this data has been collected from the Delhi editions of top four mostread Hindi newspapers and top four most-read English newspapers in India $[9,10,11]$, the model could be scaled up and appropriated to gather similar data from other regional languages (Bangla, Tamil, Malayalam, etc.) newspapers printed in India. This will give us a huge database of usable data ready to be analysed.

\section{BACKGROUND}

According to the census of 2011 , there are 19, 569 regional or local languages in India [4]. India has 29 states and 22 scheduled languages with Hindi and English being the official languages of the country. Languages in India that have the biggest number of speakers are Hindi, Bangla, Punjabi, Marathi, Gujarati, Rajasthani, Tamil, Telugu, Kannada and Malayalam. Basically, almost every state in India has its own language.

According to the New Delhi-based Indian Newspaper Society, India has 62,000 newspapers, with a staggering $90 \%$ of them in local languages. Indian news publishers are doing relatively well interms of circulation and reader base, precisely "because they have spread their wings to smaller towns", says Divya Radhakrishnan, president of TME, the media division of Mumbai-based advertising agency Rediffusion YR [16,22]. Samanth Subramanian of The National has this really interesting piece in NY Books Review in which he explains how regional languages newspaper are being preferred by the middle-class audience of India over English newspapers. In his words, "A decade or more ago, the publishers of English newspapers scorned Indian language readers, assuming that, as hundreds of millions more Indians became literate, they would turn automatically into consumers of English papers. But the steady rise in literacy rates- from $64.8 \%$ of the population in 2001 to $73 \%$ in 2011 - has had unexpected consequences. The new middle class is increasingly found in smaller towns and prefers to read in its own regional language, rather than English [11]." According to Sridhar Samu, professor of marketing at the Indian School of Business (ISB) in Hyderabad, local newspapers in the regional languages are a big hit among the people because they cover the local news, which is more relevant for these consumers than national or international news. Moreover, they cover national and international news with the perspective of how it would concern the local people [16].

With the rise of the local economy, the regional news space has grown phenomenally over the years. According to the IB ministry, the print industry in India witnessed a growth of 5.13\% in 2015-16, 


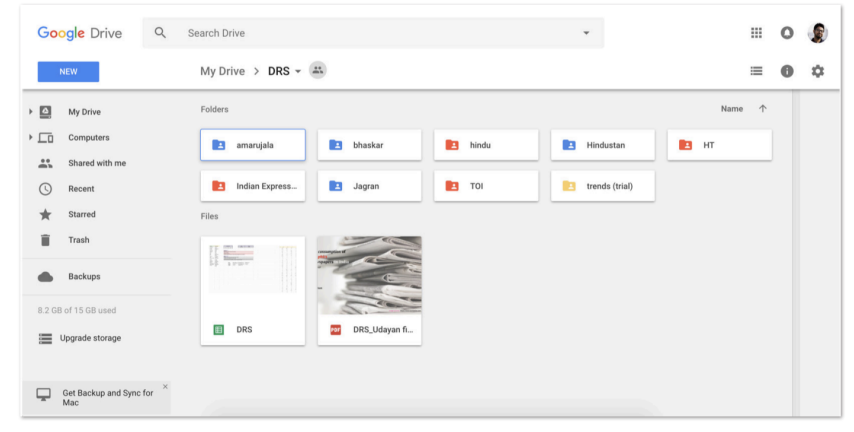

Figure 1: Collecting data: The red folders are for Hindi newspapers, blue for the English newspapers, and the yellow one is for the pilot trend analysis.

with 1,10,851 registered publications. While Hindi newspapers are overtaking the advertising-rich English language papers in terms of readership numbers, the vernacular press has broadly evolved to become the backbone of the industry. Even the report released by the central government in 2016 highlights the robust growth of the regional press. Underlining the growing importance of the vernacular newspapers, former IB Minister M. Venkaiah Naidu has gone on record to say, "Regional-language newspapers are easier to understand. We will ensure that regional newspapers claim the highest circulation in the coming years [19]."

When we showed the Tamil version of our data story on Tamil Nadu Assembly Election 2016 [21] to an audience who were readers of Tamil newspapers, they failed to understand it as they had no familiarity with infographics. We then approached the Tamil newspaper editors to ask that why don't they publish infographics in their newspapers, to which they replied that their audience was not sophisticated enough to understand infographics. This chicken-and-egg situation led to the idea of doing this project.

In the larger pursuit of collecting the infographic content of different regional languages newspapers for the same time period and comparing them across different newspapers, news, and languages, to gauge this so-called "sophistication" of the audience, this work contributes only a small part and lays a framework for the systematic collection of this type of data. We realised that there is no existing work or model to guide us in the collection of specific newspaper articles and record them with certain characteristics like layout, size, location, colour treatment, etc. Layout analysis of Indian newspaper is a challenging task to automate with code due to the presence of different font size, font styles and random placement of text and non-text regions. Hence, we resorted to a manual method of data collection which we explain in the following section.

\section{Procedure}

We started the pilot with newspapers in two languages, namely English and Hindi. We intended to compare the infographic content in the English newspapers with that present in the Hindi ones. For a fair representation of the dailies, we chose the top four most-read English newspapers in India, and the top four Hindi newspapers in India by circulation $[1,15,24]$. The English newspapers chosen were The Times of India, Hindustan Times, The Indian Express, and The Hindu. The Hindi newspapers chosen were Dainik Jagran, Dainik Bhaskar, Hindustan, and Amar Ujala. Since all Hindi newspapers were not available in Maharashtra, we went ahead with the Delhi editions in all cases to maintain regional consistency.

\subsection{Data Collection}

The data was collected from the e-paper versions of the dailies as the collection of data from the hard copy of newspapers involved a lot of manual scanning efforts. All e-papers have the exact same news content as that of their physical counterparts; only the sections containing advertisements may vary from region to region but they were not our concern. Moreover, getting hard copies of Delhi editions in Mumbai was a tough and expensive task whereas all the e-papers were easily available for use. Most newspaper websites have an e-paper archive to store papers from the previous dates $[2,3,9,10,12,13,23]$. The Hindu did not provide any access to free e-paper versions, but we found a blog that did share e-paper versions of this daily [8].

The next challenge that we faced was downloading these e-paper versions and storing them in a structured format. Most e-papers are stored as pdf of individual pages for a date. Therefore, downloading a daily with 80 pages meant navigating through each page and downloading it separately. We tried writing an algorithm for automatic downloading of each page through its URLs but we did not succeed. Each newspaper had its magazine pages attached to the main pages. These magazine pages had a different URL altogether. Since the number of main pages in each day's paper was unknown, predicting the URLs was not possible. Therefore, we could not write a script to automate the downloading and the entire process was undertaken manually. We downloaded all pages from the 31 days, from all eight newspapers and stored the pdf in date-wise folders on Google Drive [7]. A noteworthy fact is that we had trouble while downloading 'The Hindustan'. Apparently, we weren't aware that unlike the other newspapers, 'The Hindustan' did not keep an epaper in their archives for more than seven days. We missed about ten days of the newspaper there, but we made up for it by getting the hard copies of those dates.

\subsection{Renaming Conventions}

The newspaper pages in our repository needed to be renamed for proper organisation and referencing later. The renaming convention adopted was like this: (InitialsOfPaper)(DDMMYY)_(PageNumber). Once we had this huge repository of all pages of the eight newspapers collected over a month, we decided to weed out the irrelevant pages to optimise drive space. All the pages, which did not have any infographic content were deleted; we ended up retaining only about $20-30 \%$ of the downloaded content. As a tip for future attempts, we advise downloading only the pages which have relevant information, to save time and data.

\subsection{Observing trends}

Before starting to organise the data collected, we needed to know what type of data is relevant to our research, and the categories in which we could structure them. For this purpose, we conducted a test analysis over a week. All infographics from all newspapers were screenshot and populated in a google doc date-wise and newspaperwise. Throughout this week, trends in infographics were observed. These trends pertained to the type of content that was visualised, and the basic visualisation types used to make up these infographics. We observed that there were a lot of infographics in sports, crime, weather and economic section across all newspapers. Most of these infographics were in the form of bar charts, and tables. There were certain news sections like the weather and sensex, that had a fixed style of infographic content everyday. This helped us decide two important parameters for our organisation, namely, the categories of content and the visualisation types.

\subsection{Fixation of Categories}

Based on our trend observation, we divided the news content into nineteen categories. These were sports, crime, weather, environment, Sensex, economic, politics, campaign, accidents, foreign affairs, health, entertainment, technology, public opinion, education, development, administration, lifestyle, and transport. Later, as we came across different visualisations that demanded a separate category of 
their own, we added them to our classification. Two new categories that were added are history and judiciary. By the end of this project, we had twenty-one categories in total.

1. Sports: covering news related to cricket, football, Olympics, etc. The infographics are mostly related to scores, list of upcoming matches, etc.

2. Crime: covering news related to past criminal records, present criminal activities, and their repercussions. Crime stats, how a crime took place, etc., forms most of these visualisations.

3. Environment: Global events related to flora and fauna in general, like pollution and climate change, fall under this category.

4. Weather: Though the weather could have been a part of the 'environment' category, we decided to keep it separate due to its characteristic properties. Everyday temperature, climate, humidity, etc., fall into this category. Generally, this has a fixed spot in every day's newspaper.

5. Economic: News related to money, from rising and falling prices to budgets, GDP fluctuations to news related to investments, etc., fall into this category.

6. Sensex: Though Sensex could have been a part of the 'economic' category, we decided to keep it separate due to its characteristic properties. Sensex contains the daily ups and downs in the market stocks, and silver and gold prices (if printed along with the Sensex in the same space). Generally, this has a fixed spot in every day's newspaper.

7. Politics: News related to politicians, elections, crimes conducted by politicians, religious conflicts with political repercussions, etc., fall into this category.

8. Campaign: Campaigns are mostly in the form of advertisements, meant to raise awareness about a particular issue like 'Save Water', 'Save Electricity', 'Girl Child Education', etc.

9. Accidents: Any mishappening be it a road accident, suicides, or someone went missing, fall into this category. If the news also mentions that the missing person seems to have been kidnapped, then the news would fall under crime.

10. Foreign affairs: News concerning other Nations, like news regarding the foreign citizenships, development of Chabahar as a new port, etc., fall into this category.

11. Health: covering news related to diseases, health issues, hospitals, doctors, nutrition, etc.

12. Entertainment: This category includes all the news pertaining to Bollywood, Hollywood, music, museums, celebrity life events, theatres, shows, and the paparazzi news related to these events.

13. Technology: News related to the tech startups, new weapons acquired by the armed forces, upcoming smartphones and gadgets, etc., all fall into this category.

14. Public opinion: This is reserved for online polls which reflects the opinion of the people on certain issues, and news sections crowdsourced by the citizens themselves.

15. Education: News related to schools, colleges, placements, results, syllabus, coachings, literacy, etc., fall into this category.

16. Development: News related to the development of cities, services, amenities, roads, metros, technology, and their corresponding plans fall under this category.
17. Transport: News related to all forms of transport, be it metros, autos, taxis, cars, or aeroplanes, fall under this category.

18. Administration: News related to the internal functioning of the Government or and organisation fall into this category.

19. History: News related to historical events like the partition of India, Babri Masjid conflict, freedom struggle, etc., fall into this category.

20. Judiciary: News related to courts and cases, their judgements and repercussions, fall under this category.

21. Lifestyle: This was probably the vaguest category we kept. Anything that did not go into any of the other twenty categories, went in this one. This includes news like farm practices, places to go to, exercises to do, facts and miscellaneous information.

\subsection{Visualisation types}

Based on our trend observation, we identified 19 basic types of visualisations. Many infographics were complex in nature and made up of two or more of these basic 19 types. These types were namely, jumbo figures, pie chart, line chart, bar chart, bubble chart, histogram, colour, icons, table, list, ring chart, map, stacked bar chart, timeline, area chart, scatter plot, process, visual depiction of numbers, and Sankey diagram.

\subsection{Parameters of judgment}

The next challenge was organising this data by sorting it into categories. We decided upon a few parameters for a structured repository and rich data set.

1. Date: Date of the news

2. News title: This would help in identifying the news article if someone wishes to refer to it.

3. Types of visualisation: As discussed in section 4.5, the news could comprise any of the types. In case of a complex visualisation, multiple visualisation types were tabulated for the same news title.

4. Content it is depicting: A news could pertain to more than one news category as mentioned in section 4.4. We decided to stick with only the most influential news category.

5. Location: Page number on which the news appeared and the quadrant in which it appeared on that page was tabulated. More information on how we decided the quadrant and the importance of the location would be available in section 4.7.

6. Size: We needed two metrics for the size, namely the width and height of the visualisation. The width was measured in units of columns of 1.5-inch width and the height was measured in inches. More information on how we measured these metrics would be available in section 4.8 .

7. Treatment: We tabulated if the visualisation was colour or black and white, whether it was assisted with a photograph, illustration, or was presented standalone. More information on how we measured the treatment of the infographic is available in section 4.9 . 


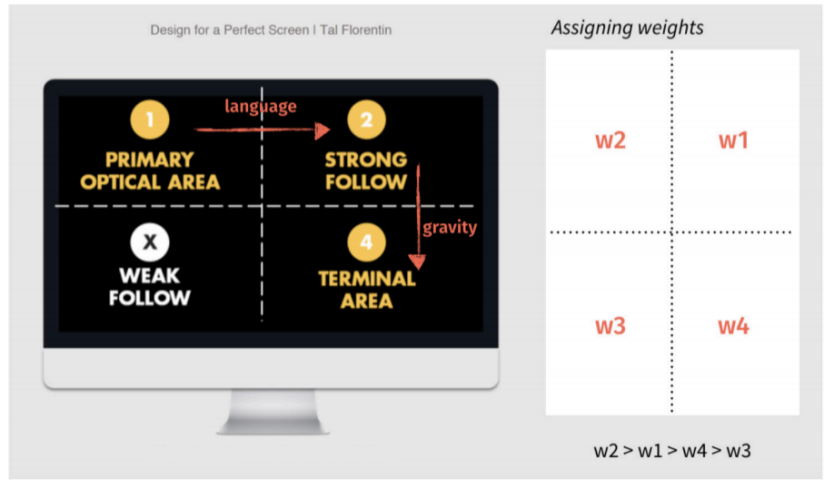

Figure 2: Quadrants and their role in the visual hierarchy

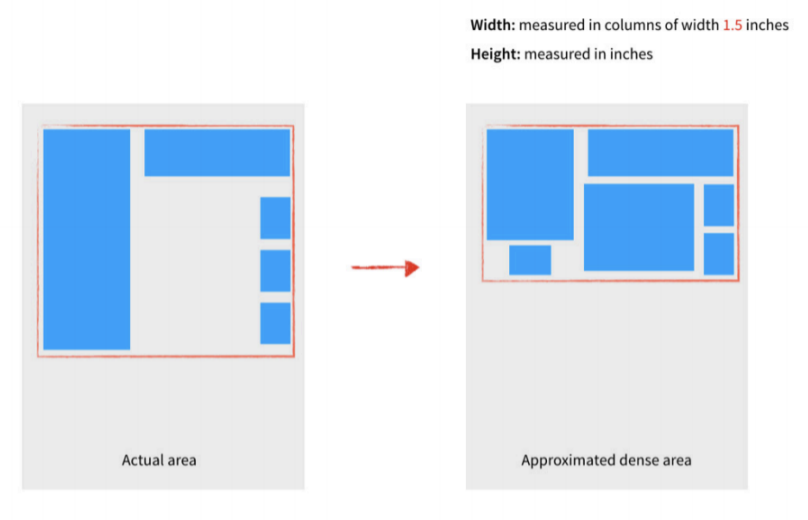

Figure 3: Approximation of dense area

\subsection{Quadrants and their role in the visual hierarchy}

In his book 'Design for a perfect screen', Tal Florentin mentions how rules of language and gravity affect gaze [5]. For English and Hindi languages, the language rule moves the gaze left to right (as the script is read from left to right) and then gravity pulls the gaze downwards (as each new line in these languages starts from below). This causes the screen to be divided into a primary optical area, an area of strong follow, terminal area, and an area of weak follow. Since a newspaper is huge compared to the normal A4 pages at work, or the A5 pages in a novel, these rules of the screen may apply here and affect gaze.

We took the quadrants as in the coordinate geometry; the toprightmost being quadrant 1 , top-leftmost being quadrant 2 , bottomleftmost being quadrant 3 and bottom-rightmost being quadrant 4 . According to this convention, quadrant 3 would be the area of weak follow while our gaze moves from the quadrant 2 to quadrant 1 and then ends on quadrant 4 (figure 2).

\subsection{Calculation of Area}

\subsubsection{Approximation of dense area}

Newspaper article layouts are quite random with different font sizes, text jutting into images randomly and images following random grids. In such cases, the area of the visualisations had to be approximated as a dense area containing only the infographic and no subject text. This approximation was done by rearranging the relevant parts into a dense rectangle (figure 3 ).

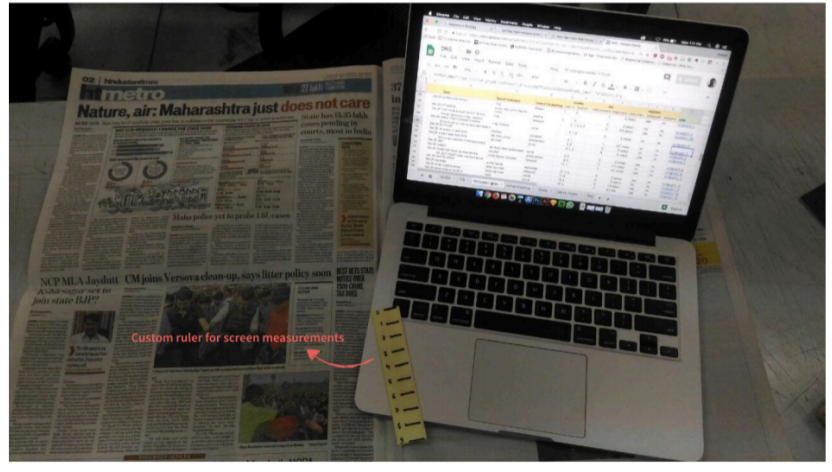

Figure 4: Measuring the size of the visualisation using a paper conversion ruler

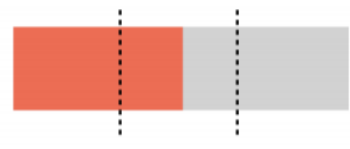

more than $1 / 3$ of total area

colour

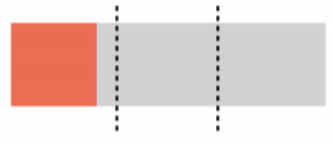

less than $1 / 3$ of total area

b\&w
Figure 5: Deciding 'colour' or 'black and white'

\subsubsection{Measuring the size of the visualisation}

Measuring the size of the visualisation from the computer screen was tricky because different laptops had different resolutions and different screen sizes. We tried making a conversion ruler out of paper by measuring the size of visualisation from the hard copy of a newspaper and from the e-paper versions. We calculated the conversion factor by finding what an inch on physical paper measured on the laptop screen. Custom grading a ruler in accordance with this conversion factor gave the same measurements on the laptop screens as they did on physical paper (figure 4). This did not work initially because all epapers had different sizes. So, in the second pass, we made separate custom rulers for each laptop and each paper to get the correct measurements.

We measured the height in inches but the width in units of columns of 1.5-inch each. This was because all newspapers conventionally have the same width and the typical eight-column layout. Measuring the width in columns made our task a little easier. We could always convert the width to inches by multiplying it by a factor of 1.5 .

\subsection{Observing the treatment of the infographic}

\subsubsection{Colour}

If the infographic's approximated dense area was coloured more than one-third of its total area, we considered the entire infographic to be coloured, as it acquired similar attention as a fully coloured infographic (figure 5).

\subsubsection{Photographs}

If the infographic was accompanied by a real-life photograph relevant to the context (figure 6), we considered a photograph to be present.

\subsubsection{Illustrations}

If the infographic was accompanied by a hand-drawn or digital illustration relevant to the context (figure 6), we considered an illustration to be present. 


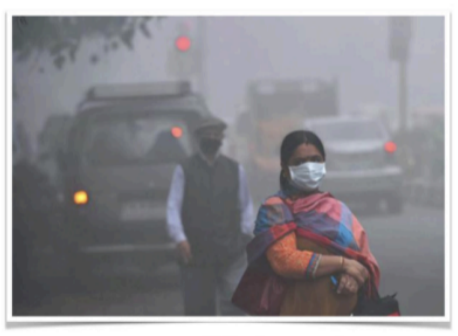

real life photograph relevant to the context

photograph

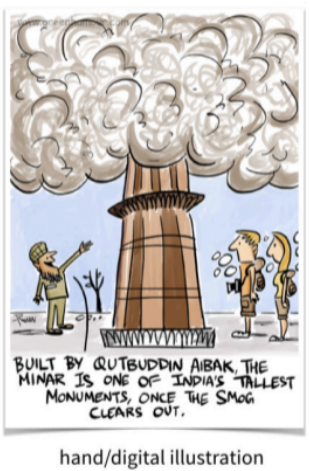

relevant to the context

illustration
Figure 6: Deciding 'photographs' and 'illustrations' (Image sources: indianexpress.com, www.greenhumour.com)

\subsection{Final dataset}

The final dataset came out to be 3002 rows x 12 columns, i.e. 36,024 cells. To ensure the correctness of the data, the dataset was verified with three different people. The link to the dataset on Google Drive [20], OSF [17] and Kaggle [14] has been given in the references.

\section{Analysis}

We pulled out some basic statistics from the collected data of various newspapers [6]. These statistics (figure 8) represent different variables, variable types and the frequency of occurrence of these variables in the newspaper (for example, we can see "what percentage of visualisations in Times of India were sports-based" or "what percentage of visualisations in Dainik Bhaskar were bar charts"). The total number of infographic observed over a span of a month was 1539 in the English newspapers and 1463 in the Hindi newspapers. Interestingly, the infographics in the English newspapers had a better colour treatment $(85.1 \%)$ than the ones in the Hindi newspapers over $(73.1 \%)$. The infographics in the Hindi newspapers had more support from the illustrations and photographs which might hint at the attempts made by the Hindi editorials to make deciphering of information easy.

Sports content was easily the most represented infographic content for both languages of newspapers. Surprisingly, the next mostrepresented content for English newspapers was the Economic category, while that of the Hindi newspapers was Lifestyle. Economic content was moderately represented in the Hindi newspapers while the Lifestyle content was poorly represented in the English newspapers. This reveals what the editorials consider to be of more interest to their readers. Politics, Weather and Environment were the next most represented content for both the newspapers.

While the English newspapers mostly used tables to represent the content, the Hindi newspapers fell back to more basic graphical elements like the jumbo figures. The English dailies also showed a fair representation through maps and ordered lists, while the Hindi dailies preferred using icons and bar chart. This is another hint that the Hindi editorials do not consider their readers to be sophisticated enough to consume complex infographics.

The Pearson and the Spearman correlation graphs at the end of each page [6] show how different variables are correlated. These statistics, however, do not tell how different variables are interdependent.

\section{ISSUES FACED AND THE SCALABILITY OF THIS METHOD}

As discussed in section 4.1, we faced issues in procuring the hard copies of Delhi editions of the selected newspapers in Mumbai as most newspapers are largely limited to a few states. We had to continue our research with the epapers which have the exact same content as the hard copies but are easily available. A flipside to these, however, is that measuring infographic content from epaper versions on a computer is tricky. The size varies according to the resolution of the computer screen used and therefore a standard resolution has to be maintained for the entire data collection. We devised a hacky method of using a custom paper scale for standardising the measurements of an electronic and a hard copy of the newspaper as explained in section 4.8.2.

Though we did not get any external validation for our collected data, we performed an internal validation session with our friends spanning three weeks. We came across several differences in opinions regarding categories and visualisation types while we were performing the internal validation of our dataset. We realised that the decided parameters are somewhat subjective and there is a need to standardise it further in future work.

This method of data collection may seem to be extensively laborious for the manual task of measuring and segregating news infographics into a spreadsheet. However, with further standardisation of the process and more human resources at work, this method seems scalable for collection of data from the other vernacular newspapers of India.

\section{Conclusion ANd Future Work}

This work explained a reproducible method of infographic data collection and organisation from the Indian newspapers. We believe that this method could work for any newspaper irrespective of its language. The collected dataset is rich enough to produce basic insights currrently as shown in section 4 . However, there is a need to develop an effective analysis tool that can convert this data into a matrix showing interdependant values. A richer comprehension of the impact of these infographics is possible only when we establish the relationship between different parameters of data. This would allow us to analyse details like "how many sports visualisations in Times of India were bar charts". Moreover, for effective analysis, it would be imperative to collect this data over a longer period of time to observe the changing trends in news and the reaction of different newspapers to the change. Last but not least, future work may also look at marking important news with codes so that we can compare how different newspapers treat the same news.

\section{ACKNOWLEDGMENTS}

The authors wish to thank Parth Kapadia, Ridhima Tapia, Supriya Dutta, and Nikhil Wani for their assistance during the analysis task and Amit Jena for feedback on the paper.

\section{REFERENCES}

[1] 10 best english newspapers in india. https://thingsinindia.in/ 10 -best-english-newspapers-in-india/.

[2] Amar ujala e-paper. https://epaper . amarujala.com/?format= img\& $=$ today.

[3] Bhaskar e-paper. https://epaper.bhaskar.com/.

[4] Census: More than 19,500 languages spoken in india as mother tongues. https://gulfnews.com/world/asia/india/ census-more-than-19500-languages-spoken-in-india-as-mother-tongue 2244791.

[5] Design for a perfect screen. https://www.webwoods.co.uk/ downloads/design-for-a-perfect-screen.pdf.

[6] Drs. https://udayanvidyanta.github.io/DRS/.

[7] Drs folder. https://drive.google.com/drive/folders/ 1t7Ptx-HJuhrZSqb5dYSP2zSDrdda9NKd.

[8] Hindu e-paper. https://shashidthakur23.wordpress.com/ newpapers/the-hindu-epaper/.

[9] Hindustan e-paper. http://epaper.livehindustan.com/.

[10] Hindustan e-paper. http://paper.hindustantimes.com/ epaper/viewer . aspx. 


\begin{tabular}{|c|c|c|c|c|c|c|c|c|c|c|c|}
\hline TOI & Hindustan_times & Hindu & Dainik_Jag & gran & nik_bhaskar & Amar_Uja & Hinc & Iustan & & & \\
\hline \multicolumn{12}{|c|}{ Show rows with cells including: } \\
\hline$\triangle$ Date & News & Type of vis... & Content it i... & page no. & quadrant & width (colu... & height (inch) & colour/ b\&w & photograph & illustration & code \\
\hline $2017-12-01 \ldots$ & Your 2-wheeler can also sport fancy number & jumbo figure... & transport & 7 & 2 & 2 & 2 & colour & yes & no & TOI011217_07 \\
\hline $2017-12-01 \ldots$ & Winter's here, but more ciites see improvement in... & table & environment & 12 & 2 & 2 & 4 & colour & no & no & TOI011217_12 \\
\hline $2017-12-01 \ldots$ & Pollution watch & bar chart, co... & environment & 5 & 2,3 & 1 & 5.8 & colour & no & no & TOI011217_05 \\
\hline $2017-12-01 \ldots$ & Pacers vs Spinners: on previous SA tour (2013-14) & table & sports & 25 & 1 & 1.5 & 2 & colour & yes & no & TOI011217_25 \\
\hline $2017-12-01 \ldots$ & Over 12000 picked to get DDA flats & map, table & development & 5 & 2 & 3 & 3.2 & colour & yes & no & TOI011217_05 \\
\hline $2017-12-01 \ldots$ & Last Gasp sterling & table & sports & 26 & 1 & 1.2 & 1.3 & colour & yes & no & TOI011217_26 \\
\hline $2017-12-01 \ldots$ & Indians most satisfied with their sex lives & pie chart, rin... & lifestyle & 7 & 1,2 & 4 & 4.7 & colour & no & yes & TOI011217_07 \\
\hline $2017-12-01 \ldots$ & Economy shakes off DeMon \& GST impact with $6 \ldots .$. & line chart & economic & 1 & 2 & 2 & 1.6 & colour & no & no & TOI011217_01 \\
\hline $2017-12-01 \ldots$ & Double deck flyover, underpass to unclog city's w... & map, icons & development & 2 & 1,2 & 4 & 3 & colour & no & no & TOI011217_02 \\
\hline $2017-12-01 \ldots$ & Desis 2nd biggest group to get US citizenship in '16 & stacked bar ... & foreign affairs & 10 & 2 & 2 & 2 & colour & no & no & TOI011217_10 \\
\hline
\end{tabular}

Figure 7: Format of the final dataset

\begin{tabular}{|l|l|l|}
\hline & English & Hindi \\
\hline Total observations & 1539 & 1463 \\
\hline \multirow{4}{*}{ Content } & sports (16\%) & sports (16.25\%) \\
\cline { 2 - 3 } & economic (11.85\%) & lifestyle (8.08\%) \\
\cline { 2 - 3 } & politics (7.3\%) & politics (5.62\%) \\
\cline { 2 - 3 } & weather (5.28\%) & weather (5.52\%) \\
\cline { 2 - 3 } & environment (4.22\%) & economic (3.8\%) \\
\cline { 2 - 3 } & & environment (2.98\%) \\
\hline \multirow{4}{*}{ Infographic type } & table (22.4\%) & jumbo figures (35.15\%) \\
\cline { 2 - 3 } & jumbo figures (9.25\%) & table (10.2\%) \\
\cline { 2 - 3 } & map (3.32\%) & icons (5.5\%) \\
\cline { 2 - 3 } & list (2.5\%) & bar chart (3.6\%) \\
\hline Colour treatment & $85.10 \%$ & $73.13 \%$ \\
\hline Illustrations & $11.50 \%$ & $15.33 \%$ \\
\hline Photograph & $42.95 \%$ & $55.78 \%$ \\
\hline
\end{tabular}

Table 1: A comparison of English and Hindi newspapers

[11] India after english? https://www.nybooks.com/daily/2014/06/ 09/india-newspapers-after-english/.

[12] Indian express e-paper. http://epaper.indianexpress.com/.

[13] Jagran e-paper. https://epaper.jagran.com/epaper/.

[14] Kaggle data. https://www.kaggle.com/nikhilwani/ regional-newspaper-visualisation-metadata.

[15] List of newspapers in india by circulation. https://en.wikipedia. org/wiki/List_of_newspapers_in_India_by_circulation.

[16] Medium for the masses: How indias local newspapers are winning rural readers. https://knowledge.wharton.upenn.edu/article/ medium-for-the-masses-how-indias-local-newspapers-are-winning-rural-readers/.

[17] Osf repository. https://osf.io/qacyz/?view_only= 266c6171cf4a4ff8ab3c5c311a096952.

[18] Picture superiority effect. https://en.wikipedia.org/wiki/ Picture_superiority_effect.

[19] Regional newspapers drive growth for print media in india: I venkat, director, eenadu. https: //www.exchange4media.com/media-print-news/ regional-newspapers-drive-growth-for-print-media-in-indiai-venkatdirectoreenadu-70196. html.

[20] Spreadsheet. https://docs.google.com/spreadsheets/d/ 1LwOEWxPAXjrXp-YjV_JiY9XrPkQHWUylpOL_LzzSZuY/edit\# gid $=1839207800$.

[21] Tamil nadu assembly elections 2016. https://info-design-lab. github.io/TN2016/.

[22] This is a list of regional newspapers in india, listing newspapers printed and published in india. http://gunjanlife.blogspot.com/2010/ 04/this-is-list-of-regional-newspapers-in.html.

[23] Timesgroup. https://epaper.timesgroup.com/Olive/ODN/

TimesofIndia/.
[24] What is the best english newspaper in india? why? https://www.quora.com/ What-is-the-best-English-newspaper-in-India-Why.

[25] J. Bullas. Awesome reasons to use infographics in you content marketing, 2015. 